\title{
MALDI imaging mass spectrometry: bridging biology and chemistry in drug development
}

Our understanding of drug tissue distribution impacts a number of areas in drug development, including: pharmacology, pharmacokinetics, safety, drug-drug interactions, transport and metabolism. Despite their extensive use, autoradiography and tissue homogenate LC-MS analysis have limitations in providing a comprehensive assessment of tissue distributions. In the case of autoradiography, it is the inability to distinguish between parent drug and drug metabolites. In LC-MS analysis of tissue homogenate, all tissue localization information is lost. The emerging technique of MALDI imaging mass spectrometry has the capability to distinguish between parent and metabolites while maintaining spatial distribution in tissues. In this article, we will review the MALDI imaging MS methodology as applied to drug development and provide examples highlighting the impact of this important technique in drug development.

A thorough understanding of absorption, distribution, metabolism, excretion and toxicity properties of drug candidates is essential in drug development and is the core focus of drug metabolism and disposition departments. Knowledge of tissue distribution is linked to many other areas in drug development including pharmacology, pharmacokinetics and drug-drug interactions [1]. For a drug to have the desired pharmacological activity it must be highly associated with the intended biological target site [2]. Secondary pharmacology and toxicity can also result from the unexpected association of parent drug or metabolites with nontargeted biological receptors, often as the result of accumulation and localization in tissues. Furthermore, drugs and metabolites are typically not homogeneously distributed in tissues, and plasma concentrations do not necessarily reflect tissue and sub-tissue compartment concentrations $[3,4]$. The benefit of tissue distribution knowledge has been demonstrated in several areas, including oncology drugs where the focus is on tumor tissue targets, and antibiotics where effective treatment is dependent on getting the active drug to the specific site of the infection $[5,6]$.

For the most part, drug tissue distribution studies and the analytical methodologies have not changed much over the past 2-3 decades despite enormous advances in many analytical areas. Most of our knowledge of drug tissue distribution is derived from single-dose whole-body autoradiography (WBA) studies in a rodent species, and in some instances tissue homogenate analysis in other preclinical species [7]. Extrapolation from these experiments is required to understand the complexity of drug tissue distribution in preclinical species as well as in humans.

Tissue imaging of drug distributions based on autoradiography or autoradioluminography relies on the presence of a radiolabeled compound, typically ${ }^{14} \mathrm{C}$ or ${ }^{3} \mathrm{H}$, to view drug-related material [8]. Because the detection method is based on the presence of a radiolabel only, there is no structural information associated with this technique. The images and quantification are strictly due to the presence of the radiolabel incorporated in the dosed drug. Thus, images broadly reflect the distribution of drug-related material while the quantification results are converted to nanogram equivalences of the parent molecule. In cases where metabolic biotransformation results in cleavage or ring scission, the radiolabel may no longer be associated with the pharmacophore portion of the parent drug. Discerning the amount of drug-related material in a tissue is valuable, but knowing whether the drug-related material is predominantly parent or consists of a large number of structurally diverse metabolites is critical in understanding drug distribution and the implications for pharmacology and safety. Despite these limitations, the good specificity for drug-related material against the background, sensitivity, quantitative nature and large dynamic range have made this approach the industry standard and accepted by regulatory authorities [9]. For most WBA studies supporting a development

\section{Stephen Castellino ${ }^{\dagger 1}$, M Reid Groseclose' \& David Wagner'}

'Department of Drug Metabolism \& Pharmacokinetics, Platform Science \& Technology, GlaxoSmithKline, 5 Moore Drive, RTP, NC 27709 USA

${ }^{\dagger}$ Author for correspondence: Tel.: + I 9194832262

Fax: + | 9194830443

E-mail: steve.x.castellino@gsk.com 


\section{Key Terms}

\section{Spatial resolution:}

Center-to-center distance

between adjacent acquisition spots (pixels) in an MALDI imaging MS experiment (defined here as: $\mathrm{m} / \mathrm{Dm}$ where $\mathrm{m}=$ mass and $\mathrm{Dm}=$ peak width at half maximum $[50 \%]$ ).

\section{MALDI (matrix-assisted} laser desorption/

ionization): Soft ionization technique used in MS that enables drugs and biomolecules to be detected without significant fragmentation.

Imaging MS: Technology used to evaluate the label-free distribution of endogenous and exogenous molecules in a tissue.

Histology: Microscopic anatomical evaluation of thin tissue sections and a critical aspect in interpreting images generated by MALDI imaging MS . program, a time profile study design is employed in order to follow the distribution and amounts of drug-related material as a function of time after dosing. This study design provides the kinetics associated with life time of drug-related material in specific tissues. Typical spatial resolution in WBA studies is between 50 and $100 \mu \mathrm{m}$; however, spatial resolution as low as $10 \mu \mathrm{m}$ is possible with micro-autoradiography studies [10]. Micro-autoradiography studies are technically more challenging and not strictly quantitative and, therefore, are not routinely used in the pharmaceutical industry.

The other common method for establishing drug tissue distribution is through LC-MS analysis of tissue homogenates. Results from this approach are typically reported in nanograms of drug or metabolite per gram of tissue. The value of determining tissue concentrations by LC-MS analysis of tissue homogenate has been debated for years [2,5]. Interpretation and extrapolation of these data can be very tenuous because the data provide no distribution information within a specific tissue or organ, and in essence, treat all tissues as homogeneous components, ignoring the distinct compartments and functions within tissues and organs. This method structurally discriminates between parent and metabolites and is quantitative, provided appropriate methods are employed.

MALDI imaging MS (IMS) provides the opportunity to determine discreet tissue localization for parent drug as well as metabolites [11-13]. Combined with strategies to address quantification, this approach can offer significant advantages over autoradiography methods and tissue homogenate analysis for determining drug and metabolite tissue distribution in a preclinical setting. Recent advances in commercially available instrumentation ranging from matrix application tools, matrix-assisted laser desorption/ionization (MALDI) imaging-centric mass spectrometers and software are providing a pathway to mainstream this approach beyond the laboratories of the pioneers in this area. This is an emerging and rapidly growing field with demonstrated impact in areas outside of drug development such as proteomics and lipidomics. However, recent questions have been raised about the readiness of MALDI IMS technology to be broadly used in drug research [14]. Specifically, the impact and understanding associated with sample preparation, ion suppression and quantification will be critical for mainstream applications in all areas, including drug distribution studies.

\section{Overview of MALDI IMS}

MALDI IMS has emerged as a powerful and diverse technology for analyzing the spatial distribution of endogenous and exogenous compounds directly from a tissue section [15-18]. This review will focus mainly on the application of MALDI IMS for the analysis of tissue samples. Several recent reviews provide a broad overview of other MS imaging methods and applications [19-25]. These include alternative ionization techniques such as secondary ion MS (SIMS), desorption electrospray ionization (DESI) and hybrid methods such as MALDI electrospray ionization (MALDESI).

In short, a typical MALDI IMS experiment is conducted by coating a thin tissue section mounted onto a target with an appropriate matrix solution. This solution serves to extract analytes of interest from the underlying tissue and upon solvent evaporation the extracted molecules are co-crystallized with the matrix. The role of the matrix is to absorb the laser energy and facilitate desorption/ionization of the analyte molecules [26]. Mass spectra are then acquired across the tissue at defined geometrical coordinates. The resulting dataset contains hundreds to thousands of individual spectra consisting of all ions detected at each location of acquisition. Custom software is then used to compile the mass spectra into a format where each spectrum represents a discrete pixel and the distribution and intensity of any of the detected species can be viewed across the tissue as an ion density map or image (FIGURE I).

In a single MALDI IMS experiment it is possible to detect hundreds or even thousands of discrete signals across a tissue from a diverse set of analytes. These analytes can range from endogenous biomolecules (e.g., proteins, peptides and lipids) to exogenous molecules (e.g., pharmaceutical compounds). Thus, one of the major advantages of using this technique is that endogenous biomolecules are also automatically detected, providing opportunities to evaluate the underlying physiological state of a tissue [27]. There are several aspects of imaging MS that merit critical evaluation and will be discussed in detail, including sample preparation, ion suppression, sensitivity and data analysis.

\section{Sample preparation}

As with many analytical methods, a successful outcome is closely aligned with the rigor and attention paid to sample preparation steps. MALDI IMS sample preparation is unique in that most practicing mass spectrometrists are 


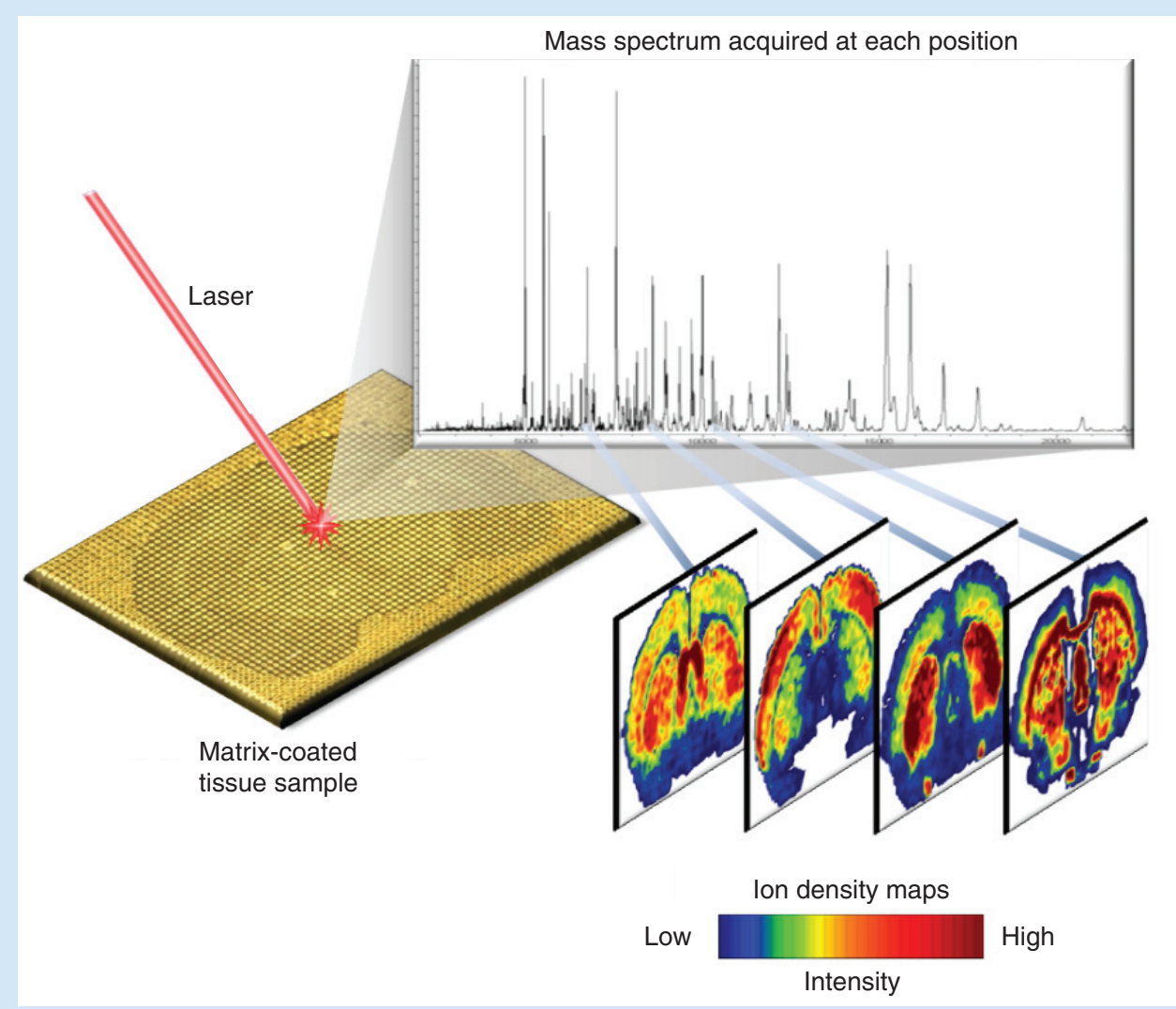

Figure 1. MALDI imaging mass spectrometer experimental workflow. The MALDI imaging experiment is initiated by mounting a tissue section onto a target, applying a matrix solution and rastering a laser across the surface of the tissue. At each discrete location where the laser is fired, a mass spectrum is acquired. By plotting the ion intensities as a function of the $x$ and $y$ coordinates on the tissue, ion images are generated.

typically not skilled in the art of tissue sectioning with a cryostat. Thus, many spectrometrists will require assistance in this process from histologists or toxicologists. Typically, freshfrozen tissues are sectioned at $10-12 \mu \mathrm{m}$. The frozen sections are then thaw mounted onto a MALDI target prior to desiccation and matrix application.

For whole body IMS experiments, preparation of tissue sections is different because the sample specimen (i.e., rat) is typically embedded in a block of ice or carboxymethylcellulose. Thicker sections $(20-50 \mu \mathrm{m})$ are collected on a whole-body cryostat and then mounted onto the MALDI target with adhesive tape.

Although fresh-frozen tissues are ideal, the analysis of formalin-fixed, paraffin-embedded tissues using MALDI IMS has been reported for proteins and peptides [28,29] and also for small molecules [30]. However, due to the limited number of publications in this area, the compatibility and utility of these fixed tissues for drug analysis with MALDI IMS remains unclear. It is likely that the formalin fixation process, where a tissue is placed into a formalin solution for several hours, as well as other histology processes such as alcohol dehydration and paraffin infiltration, will result in diffusion or even extraction of some drug compounds, rendering the tissue unusable for drug imaging experiments. In addition, the fixation process, which forms methylene bridges between the primary amines of lysine on proteins, may detrimentally affect the extractability of drug compounds. It should be noted that the vast archives of formalin-fixed, paraffin-embedded tissues available at most pharmaceutical companies could represent an extremely valuable source of samples if these technical difficulties could be overcome. Embedding media that do not interfere with the MALDI process have recently been reported and can be useful in aiding cryosectioning of certain types of tissue samples [31]. 


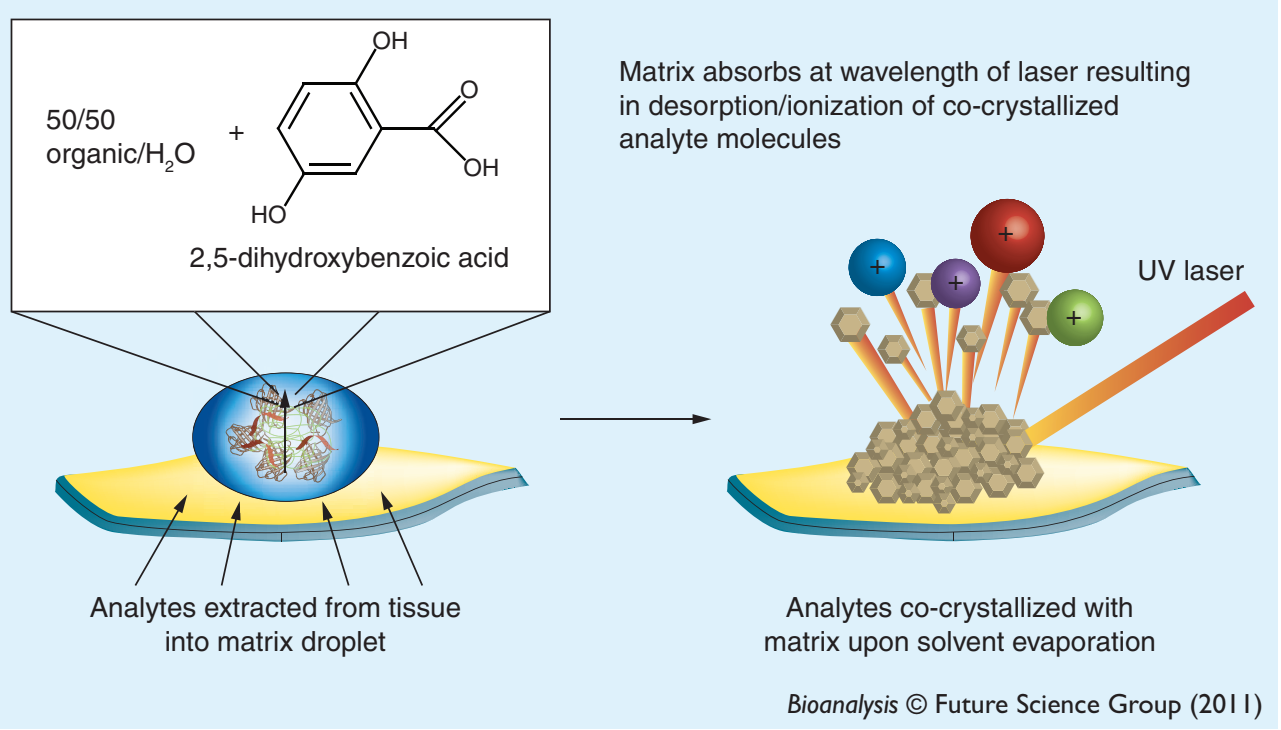

Figure 2. Role of matrix application in MALDI imaging.

A matrix solution when applied to the tissue section serves to extract analytes of interest from the underlying tissue, and upon solvent evaporation the extracted molecules are co-crystallized with the matrix to facilitate subsequent MALDI MS analysis (Figure 2). The selection of the matrix solution and method of application is primarily driven by empirical results. For small organic

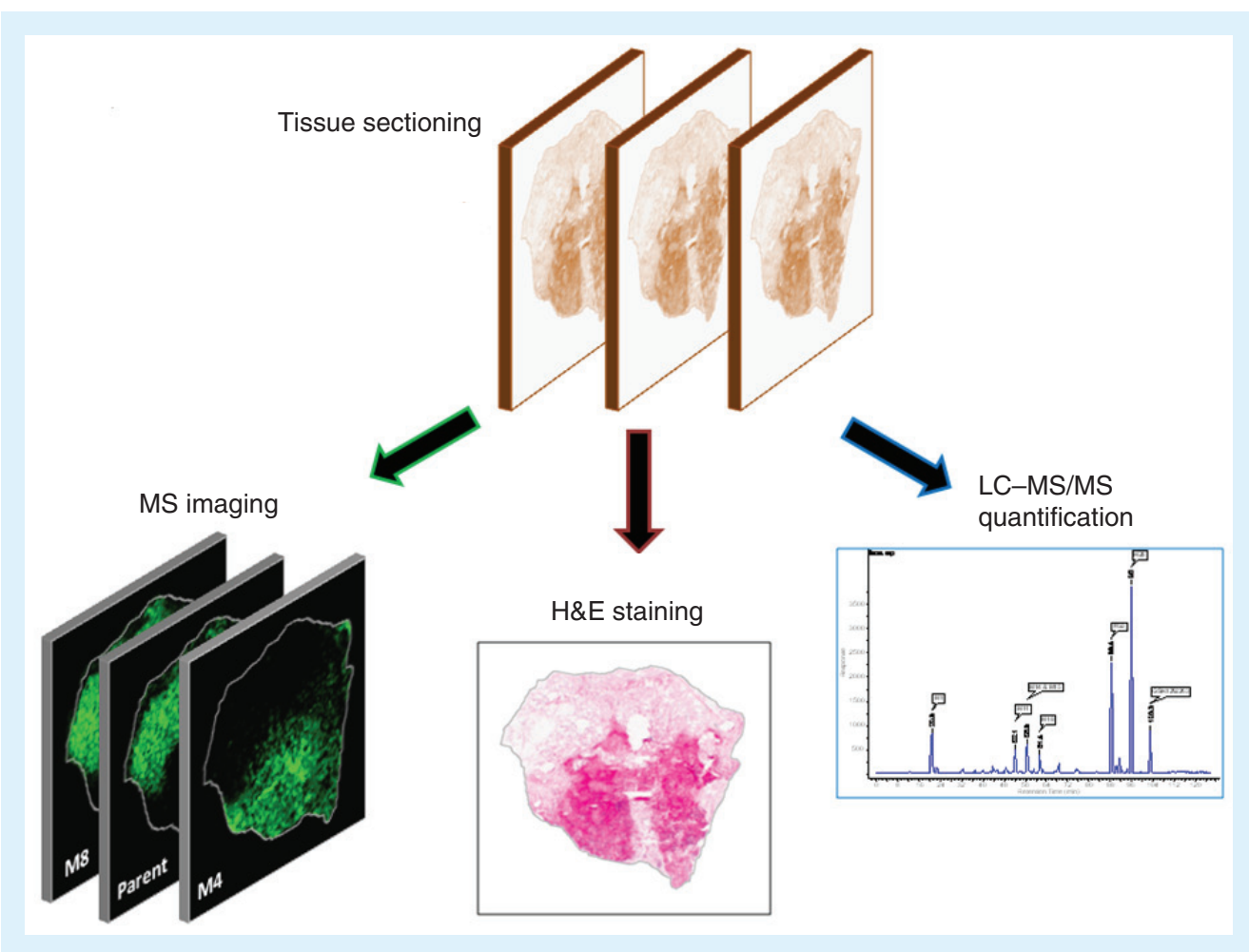

Figure 3. Serial sectioning strategy for correlating ion images, histology and LC-MS quantification. By taking serial sections from tissue for MALDI imaging, H\&E staining and LC-MS quantification, correlations between drug distribution, histology and drug quantification will be improved. Drug and metabolite concentrations determined from whole organ analysis may not be representative of sections used for MALDI imaging. H\&E: Hematoxylin and eosin. 
molecules the most commonly reported matrix compounds are 2,5-dihydroxybenzoic acid, $\alpha$-cyano-4-hydroxycinnamic acid and picolinic acid. As a starting point, the optimum matrix is typically determined by spotting drug standard onto the MALDI target and observing which matrix provides the maximum $S / N$. Several other factors, including undesired analyte fragmentation, inhomogeneous crystallization and excessive matrix background peaks may also play a role in choosing a desirable matrix. In our laboratory, 2,5-dihydroxybenzoic acid tends to be the matrix of choice for the analysis of small molecules by MALDI IMS. The matrix is usually dissolved in an acidified mixture of organic solvent(s) and water (i.e., 50/50 methanol/water and $0.1 \%$ trifluoroacetic acid). The matrix solution has two critical functions: extraction of analytes of interest from the tissue and co-crystallization of the analytes with the matrix to facilitate the desorption and ionization processes. Discussion of the fundamentals of the MALDI process is beyond the scope of this review, but additional information can be found in literature focused on this topic $[26,32-38]$.

In theory, the choice of solvent composition could be guided by the physicochemical properties of the analyte or analyte class of interest where more polar analytes may prefer higher percentage aqueous and nonpolar analytes a higher percentage of organic solvent. While this is an important consideration, this parameter is complicated by several additional factors, including the complexity of the biomolecular interactions in a tissue, matrix solubility, and the solvent evaporation/ matrix crystallization process [21]. As a result, selection of an optimal solvent composition may be determined empirically in many cases.

The final step of sample preparation is the application of the matrix solution to the targetmounted tissue. A variety of matrix application methods have been successfully used, including microspotting [39], sublimation [40,41] and spray coating [42]. One of the key considerations in selecting an application method is the reproducibility of the matrix application so that a number of tissues can be analyzed with minimum variability from the matrix application. Several automated systems have been developed and marketed including the HTX Imaging ${ }^{\mathrm{TM}}$ sprayer (nozzle spraying technology) [101], Bruker Image Prep $^{\mathrm{TM}}$ (vibrational vaporization) [102], Shimadzu CHIP-1000 (inkjet printing technology) [103], and Labcyte Portrait ${ }^{\circledR}$ (acoustic droplet technology [104]. These systems permit accurate control of application variables, such as coating cycles, drying times and matrix thickness, and therefore will provide increased reproducibility and optimization relative to manual application. In general, imaging from tissues with high analyte concentrations and acquisition at low spatial resolution will be less demanding on the optimization of matrix solution selection and application method.

\section{Ion suppression, sensitivity \& quantification}

As is the case for any application of MS, the issues of ion suppression, sensitivity and quantification are important interrelated variables that need to be evaluated and addressed. In MALDI IMS, reproducibility and consistency in analyte extraction and matrix co-crystallization between areas of a tissue containing different cell types or structural morphologies are of greatest concern. Many of the questions surrounding the extent of ion suppression in tissue imaging have been discussed extensively in the literature $[14,43]$.

The effect of ion suppression is observed when comparing the signal intensity of a standard spotted directly on the MALDI target versus the signal from the same amount spotted on the surface of a control tissue section $[13,14]$. Typically, this experiment reveals a discrepancy in the observed signal for equal amounts of an analyte where signal intensities are significantly higher when acquired directly from the target. Multiple factors are likely to be contributing to the difference in observed signal including variation in matrix crystallization, ion suppression from endogenous salts and lipids, and molecular interactions between the analyte and tissue biomolecules. In an imaging experiment, where interpretation of the ion density map for an analyte is based on differences in intensity and is therefore fundamentally quantitative, it is important to establish that the variation of signal intensity across the tissue reflects the actual concentration and is not governed by the effects of ion suppression.

One strategy to evaluate ion suppression is to uniformly coat the surface of a control tissue section with a standard and acquire the MALDI image $[1,44]$. Ideally, this experiment would identify any regions of a tissue section that may introduce significant ion suppression effects on the analyte standard by showing deviations from a homogenous distribution. However, accurate assessment of ion suppression requires careful experimental design and it is critical that both the standard and the matrix solution are applied homogenously and reproducibly across the tissue section. 
Another way to address the potential impact of ion suppression is to use a post-acquisition normalization of spectral intensities. Normalization of the data in an IMS experiment is carried out by multiplying all spectra by an intensity scaling factor. This scaling factor can be determined by a number of different methods (i.e., total ion current), each having advantages and disadvantages; several recent publications have discussed this topic in detail [45-47].

In the context of structurally characterizing drug metabolites from biological matrices, inevitably the issue of quantification will always be raised. This is because pharmacology and toxicology are driven by knowledge of both the inherent reactivity of a molecule and the quantity at the receptor site. One strategy is to use quantitative LC-MS data from tissue homogenates to try and correlate drug quantities with the intensities detected in an ion image [48]. This strategy assumes that tissues are homogenous and typically utilizes a large piece of tissue that may not be representative of the thin section used in the imaging experiment. Most tissues contain sub-compartments, which may contain high concentrations of particular metabolites. Consequently, the homogenate results may introduce a bias. Alternatively, serial sectioning with one section going to quantitative LC-MS and the other being used for imaging can reduce the likelihood of this bias (FIGURE 3) [49].

IMS studies employing radiolabels can take advantage of quantification through autoradiography in order to optimize both tissue distribution and quantification [50]. Alternatively, spotting standards on control tissue prior to matrix application to generate calibration curves has also been used for IMS quantification [1]. This methodology mimics the interaction of the tissue, analytes and matrix, but may not be completely representative of the tissue extraction process. These recent literature reports are promising and effectively show that a reasonable level of quantification can be obtained; however, significant improvements will be required to reach the validation level of a quantitative LC-MS assay or autoradiography. The issue of quantification in MALDI IMS represents several unique challenges and should continue to draw attention and become more refined as greater insights into the MALDI process are gained.

Evaluating limits of detection in IMS requires rethinking the definition of 'sample'. It is clear in tissue homogenate work that homogenization and extraction followed by quantitative LC-MS analysis results in an analyte amount per volume of extract that can then be converted to a tissue concentration in $\mu \mathrm{g}$ per gram of tissue. However, in IMS the entire tissue section represents an array of samples, where the sample is defined as the individual area of tissue (pixel) where a MALDI mass spectrum is acquired. Therefore, it is important to define a limit of detection (LOD) in this small volume of tissue because pixel intensities below this value will be registered as containing no drug (FIGURE 4). One immediate consequence of this is that greater sensitivity, LOD, will usually be obtained with low spatial resolution (large laser spot or averaged raster area) compared with high spatial resolution (small laser spot or averaged raster area) because the amount of analyte will be proportionally higher in a larger area. Thus, in most cases the tradeoffs between signal intensity and spatial resolution must be taken into consideration when planning an experimental strategy.

\section{Spectral resolution}

Structural characterization of metabolites from biological matrices using MS is typically carried out by employing a chromatographic separation method, such as HPLC or UPLC, prior to introducing the sample into the mass spectrometer. The chromatographic separation adds another dimension of analyte differentiation for complex mixtures prior to mass spectrometric analysis. The development of high-resolution mass spectrometers [51-54] and data-mining software such as mass-defect filtering [55-57] has further facilitated metabolite identification in biological matrices. MALDI IMS does not have the benefit of the initial chromatographic separation and the burden of resolving all components depends on the mass analyzer. This is especially challenging in the low mass range $(<1000 \mathrm{Da})$ where matrix background and high levels of endogenous species can significantly interfere with signals for drugs and metabolites. Spectrometers with relatively low spectral resolution, including quadrupoles [58], ion traps [30] and time-of-flight [27] systems can be employed in IMS, but these systems, may require MS/MS methods to provide enough specificity to overcome interference from the matrix and endogenous compounds. The analyte selectivity of MS/MS methods significantly narrows the scope of the IMS experiment to a single target molecule of interest, and therefore excludes additional drug-related material or endogenous components from detection. 


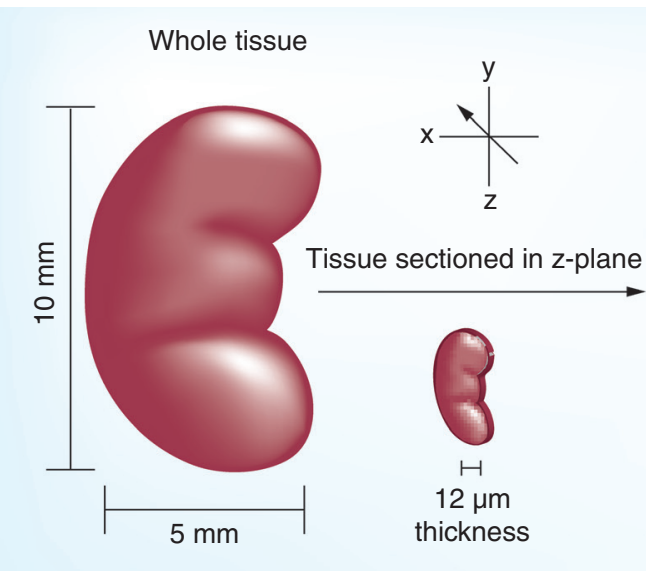

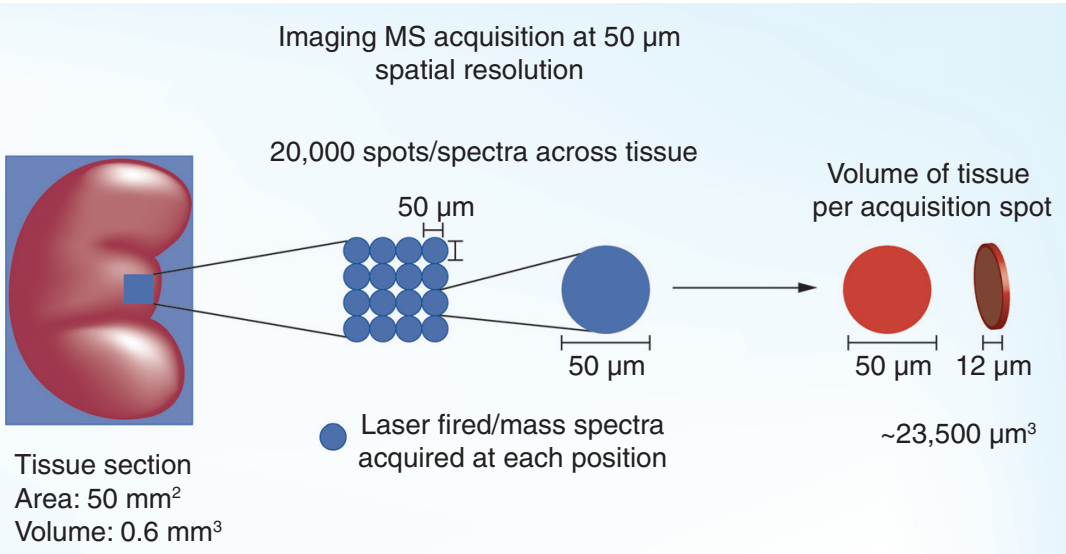

Tissue homogenate concentration: $50 \mathrm{ng} / \mathrm{mg}$
Quantity per acquisition spot $1 \mathrm{fg}$

Figure 4. Limit of detection defined by MALDI pixel size.

However, it has recently been shown that a continuous raster imaging mode where multiple ion transitions are monitored sequentially can significantly enhance the scope of this type of imaging experiment [58,59].

High-resolution (HR) mass spectrometers such as the Fourier transform ion cyclotron resonance (FT-ICR or FT) and Orbitrap have the ability to mass spectrally resolve drug and metabolites from endogenous compounds with the same nominal mass. Consequently, HR-mass spectrometer instruments can perform IMS experiments in full scan mode allowing the simultaneous detection of drug, metabolites and potential biomarkers in one imaging experiment $[1,60]$. However, the acquisition times of these instruments are typically much slower than lower resolution instrumentation, significantly increasing the total time for acquiring an image.

Several examples illustrate the importance of high spectral resolution in drug distribution studies. Figure 5 shows a region of the average spectrum and the corresponding ion images for two metabolites from a MALDI IMS experiment conducted on liver tissue from a dog dosed with lapatinib. A resolution of $>50 \mathrm{~K}$ is required to completely resolve these two metabolites in a full scan mass spectrum and confidently identify their corresponding tissue distributions. Another example of the importance of mass resolution is captured in Figure 6. Here, four singly charged ions that span a range of $0.25 \mathrm{Da}$ have been mapped as individual components and as a composite, simulating what the image would look like if the ions were not adequately resolved. The ability to resolve drugrelated peaks from background allows one to comprehensively evaluate drug tissue distributions in a single MALDI IMS experiment. It is important to note that in some cases (e.g., isobaric metabolites), MS/MS will be required to confirm a metabolite's identity.

As a general strategy, instruments with less resolving power and mass accuracy can be employed successfully in IMS if there is a single molecular species that is being targeted and MS/MS spectra can reliably provide sufficient resolution from endogenous components. Such a strategy might be well suited in a discovery setting where the goal is to image the potential drug against a biological target. However, when the goal is to understand the tissue distribution of a drug and its metabolites, instruments with high resolution and high mass accuracy can be essential for IMS studies. A relatively new type of mass spectrometer, which incorporates postionization separation based on ion mobility prior to detection has the ability to resolve even isobaric molecular components of a tissue in the gas phase based on collision cross section and, therefore, has great potential for drug imaging studies [61-64].

\section{Spatial resolution}

MALDI imaging generates 2D maps of analyte intensities across the surface of a tissue. The term spatial resolution, or pixel resolution, generally refers to the center-to-center distance between two adjacent areas of acquisition (pixels). In this review we will use the terms spatial 


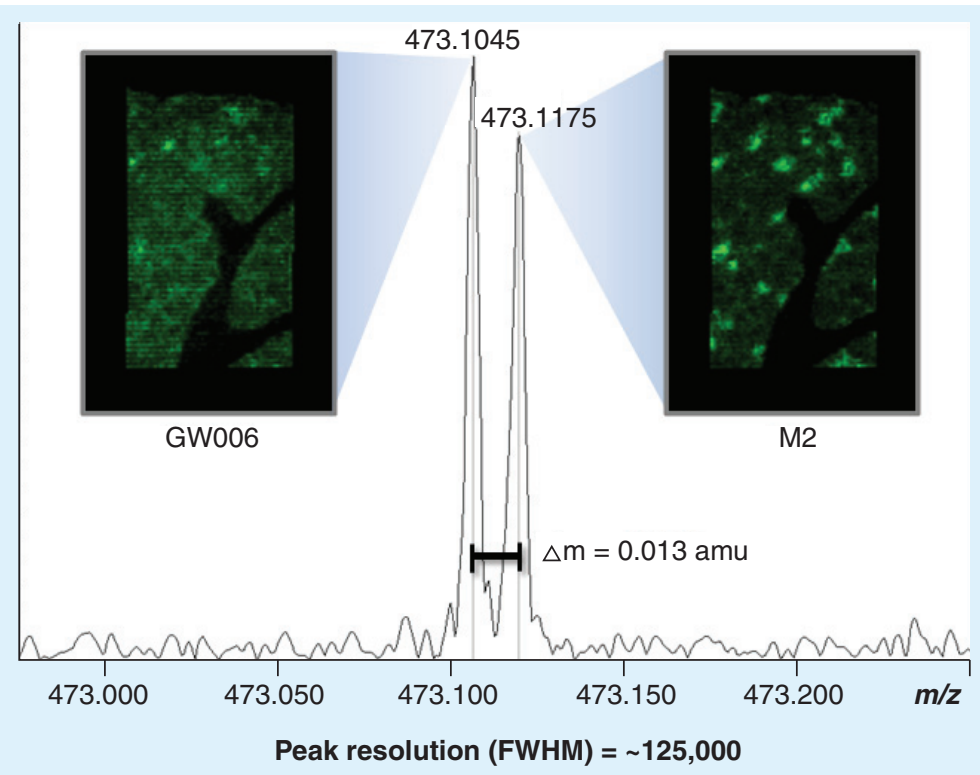

Figure 5. Two metabolites (GW006 and M2) of lapatinib from dog liver resolved by Fourier transform ion cyclotron resonance.

resolution and lateral resolution interchangeably to describe the effective laser spot or pixel of the tissue image.
Spatial resolution is one of the most important parameters that defines the utility and scope of MALDI imaging as applied to tissue distribution studies in drug development. In commercial instruments, the physical size of the laser spot is limited to approximately $10 \mu \mathrm{m}$ with most experiments using a laser spot size between 50 and $250 \mu \mathrm{m}$. Recently, smaller laser spot sizes have been achieved in custom optical systems used in IMS experiments and reveal the potential for further development in the area of high-resolution imaging $[60,65]$. The physical limitation on the choice of pixel size in an IMS experiment is determined by the laser spot size; however, several additional factors, including sample preparation, analyte tissue concentration, MALDI ionization efficiency and heterogeneity of the tissue, are also important considerations. An oversampling method can be used to achieve spatial resolutions higher than the laser spot diameter. This is conducted by using a raster increment that is smaller than the width of the laser beam and, therefore, only a fraction of the beam is ablating matrix and generating the measured analyte signal [66].

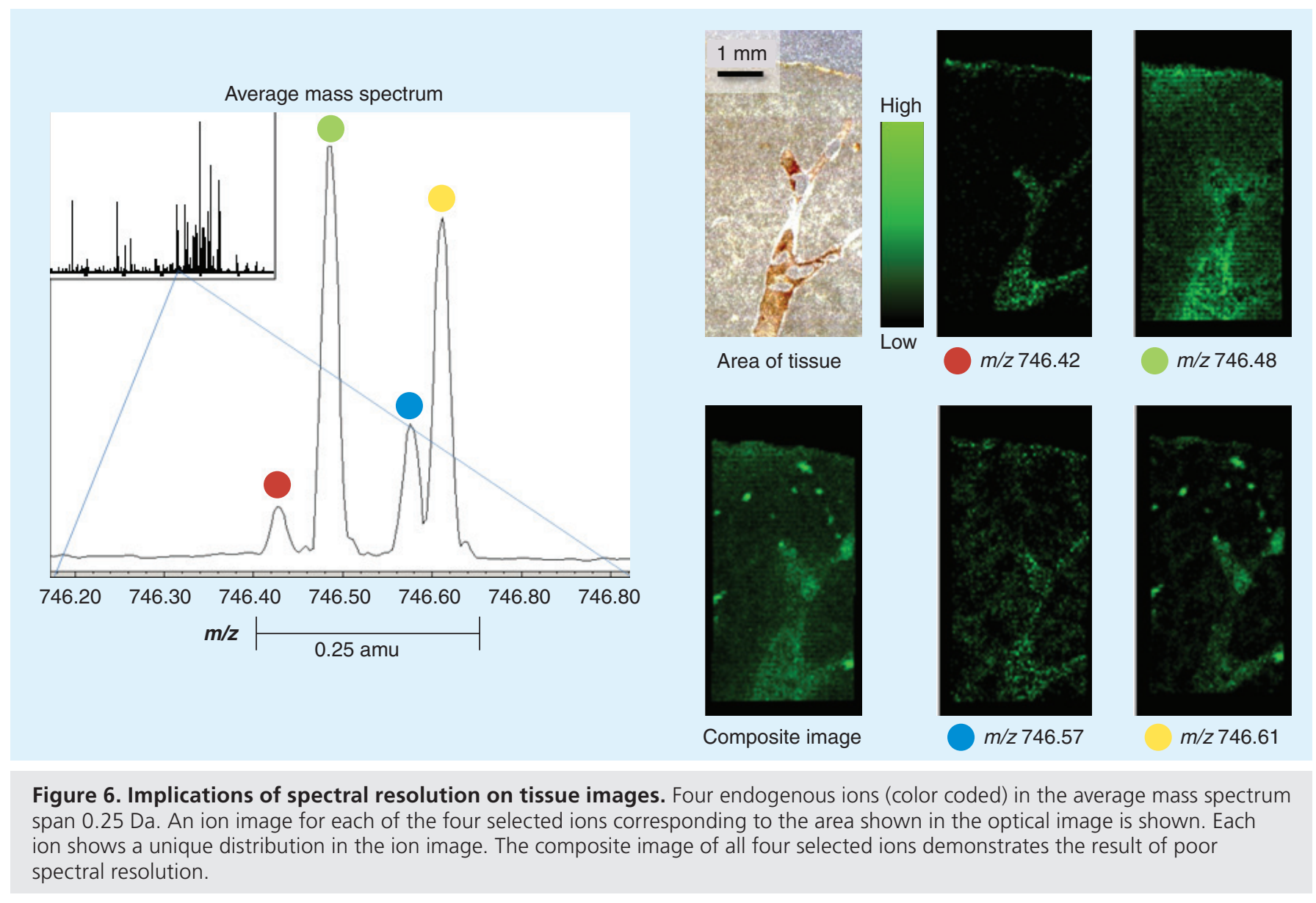


In practice, achieving higher spatial resolution can be technically challenging and time consuming. Therefore it is important to consider the minimum resolution needed to answer the question at hand prior to analyzing a sample. Tissue sampling at $100-200 \mu \mathrm{m}$ provides useful images on the order of what would be expected from WBA and can provide valuable distribution surveys of large tissue sections. Sampling with larger laser spot sizes has the advantage of speed (area sampled/time) and sensitivity (averaging spectra over a larger area). However, in some cases where analyte distributions are highly localized to specific histological features (e.g., liver bile ducts) then higher spatial resolutions $(<50 \mu \mathrm{m})$ may be required.

In some cases the resolution needed to acquire meaningful data is dependent on the type of preclinical species used in a study assuming that the tissue of interest scales proportionally with the size of the animal. For example, brain tissues are structurally heterogeneous with numerous well-defined anatomical regions of varying size and distribution. Depending on the scope of the IMS analysis, careful consideration should be taken in devising a sectioning strategy for brain tissue, especially if it is desired to have specific regions with a greater exposure in the tissue sectioning plane. FIGURE 7 shows sagittal brain sections from a rabbit after repeat dosing of a developmental drug analyzed by MALDI IMS in our lab [Castellino S, Groseclose Mr, Wagner D,
Unpublished Data]. Data were acquired on a Bruker Solarix FT-ICR MS using $25 \mathrm{mg} / \mathrm{ml}$ DHB, methanol:water:0.5\%TFA, and applied with a Bruker ImagePrep. The localization of a cysteine conjugate metabolite in the white matter of the brain is clearly visible in FIGURE 7A \& B using a spatial resolution of $170 \mu \mathrm{m}$. In a preclinical species, such as a mouse, where the size of the brain is significantly smaller a spatial resolution of $170 \mu \mathrm{m}$ would not provide the same level of detail and may not be sufficient in distinguishing many of the brain substructures.

In tissues such as kidney and liver, higher spatial resolution is required if analysis of the drug distribution into tissue sub-compartments is desired. Figure 8 displays the ion image for lapatinib and its metabolite M10 in a dog liver section at a spatial resolution of $150 \mu \mathrm{m}$ after repeat dosing at $360 \mathrm{mg} / \mathrm{kg}$ for 5 days [Castellino S, Groseclose Mr, Wagner D, Unpublished Data]. Lapatinib is highly metabolized by the liver and, in addition to parent and M10, ion images for 21 metabolites were also generated in the liver sections (data not shown). At a spatial resolution of $50 \mu \mathrm{m}$, differences between the parent and some metabolite distributions become prominent (FIGURE 9B \& C). Increasing the spatial resolution of the IMS analysis to $50 \mu \mathrm{m}$ provides a ninefold increase in data points and provides a significant enhancement in the clarity of the observed ion distributions. Increasing the spatial resolution to $35 \mu \mathrm{m}$
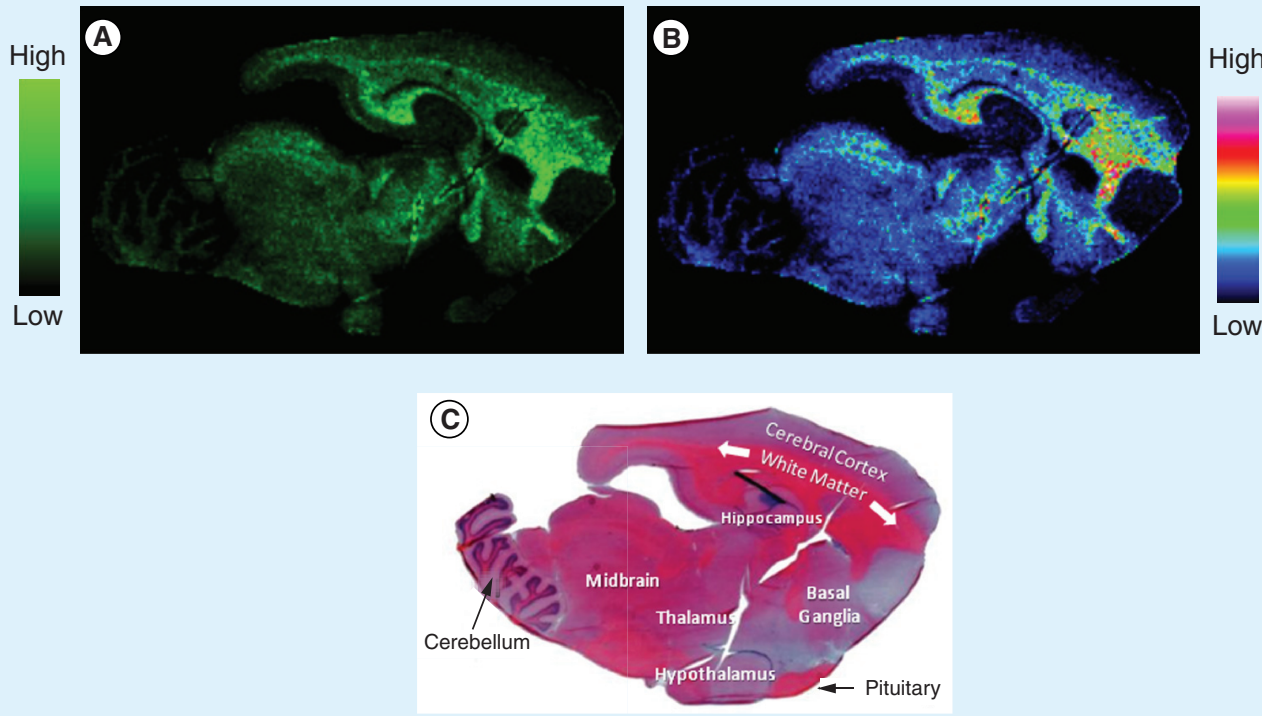

Figure 7. Sagittal rabbit brain section showing distribution of cysteine conjugate metabolite. (A) Monochromatic ion image showing brain distribution of cysteine conjugate metabolite (170 $\mu \mathrm{m}$ spatial resolution). (B) Same ion image shown in rainbow intensity format. (C) Hematoxylin and eosin histology slide showing regions of the rabbit brain. 


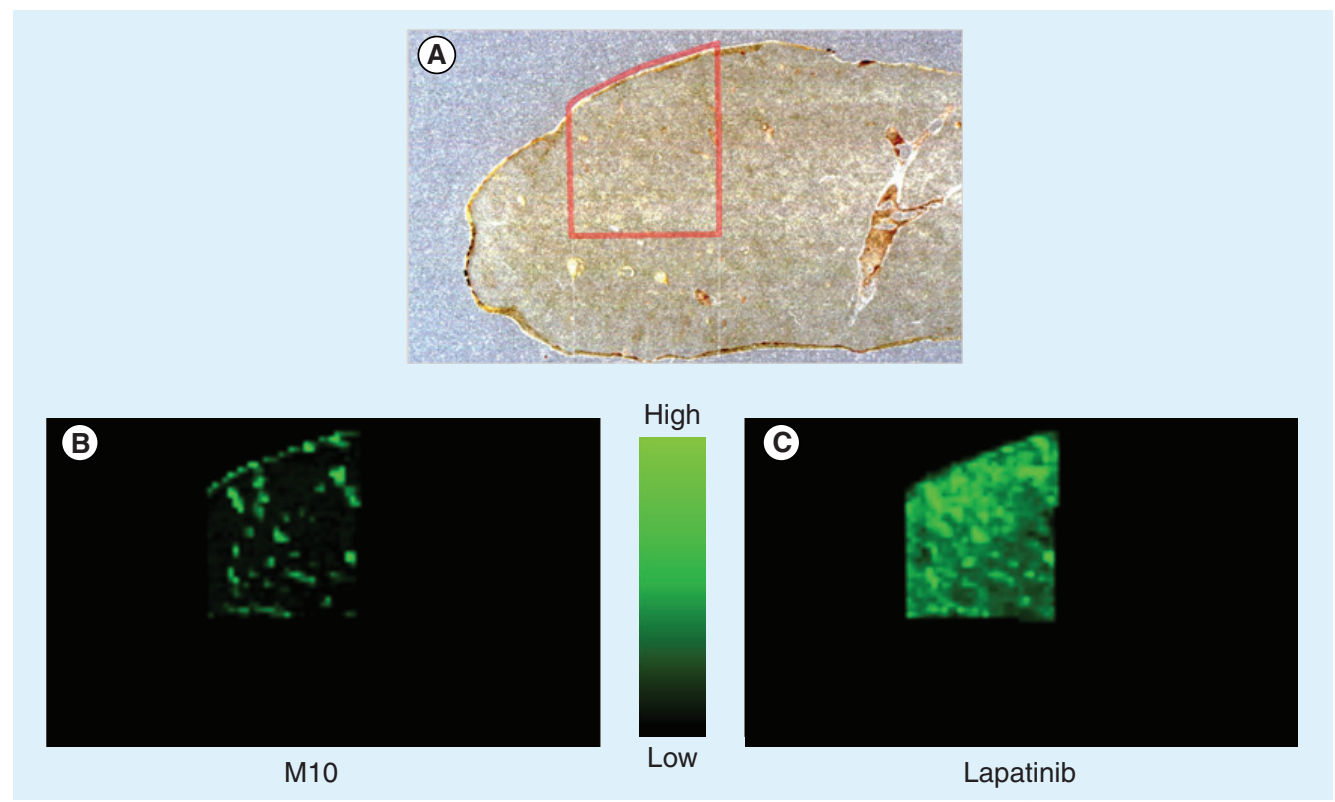

Figure 8. Ion image of a dog liver section at a spatial resolution of $150 \mu \mathrm{m}$. (A) Optical image of liver section showing area of tissue where MALDI image was acquired.

(B) Monochromatic ion image of metabolite M10, $\mathrm{m} / \mathrm{z}$ 649.14. (C) Monochromatic ion image of lapatinib, $\mathrm{m} / \mathrm{z}$ 581.14.

reveals even further refinement to the point where accurate correlation of the ion distributions with the underlying tissue histology is possible. For example, Figure 10 shows a metabolite distribution localized predominately in the small bile ductules, which are typically between 50 and $100 \mu \mathrm{m}$ in diameter. Conducting IMS experiments at this level of spatial resolution generates discreet metabolite distributions into the subcompartments of a liver tissue such as the biliary tree, portal and central veins. Tissue sections serial to those analyzed by IMS can be treated with histology stains such as hematoxylin and eosin to identify various subcompartments and enable correlation between the tissue histology and the detected ion distributions. In FIGURE II, inflammation in the histopathology sections are correlated with metabolite M10 tissue distribution.

In a similar fashion, differentiated subcompartment distributions of two drug metabolites are shown in the mouse kidney at the $2 \mathrm{~h}$ time point after 5 day repeat dosing of a developmental drug (Figure I2) [Castellino S, Groseclose Mr, Wagner D, Unpublished Data]. Note the correlation of metabolite distribution and histology of the tissue. In this study, metabolites are differentially distributed between the medulla and cortex at $2 \mathrm{~h}$ after the last dose. Rompp et al. recently showed that at $10 \mu \mathrm{m}$ spatial
(A)

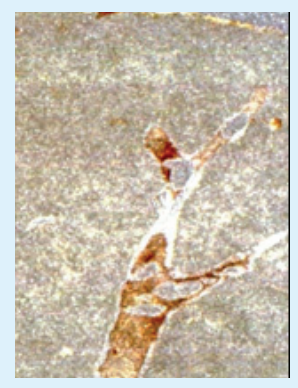

Optical image of region analyzed
(B)

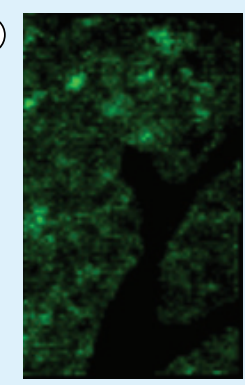

$m / z 581.14$

Lapatinib
(C)

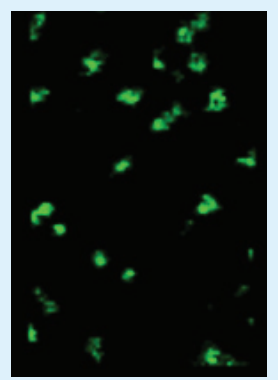

$m / z 649.14$ M10

Figure 9. Optical and ion images of a dog liver section at a spatial resolution of $\mathbf{5 0} \boldsymbol{\mu m}$. (A) Optical image of liver section. (B) Monochromatic ion image of lapatinib, $\mathrm{m} / \mathrm{z}$ 581.14. (C) Monochromatic ion image of metabolite M10, $\mathrm{m} / \mathrm{z} 649.1$. 


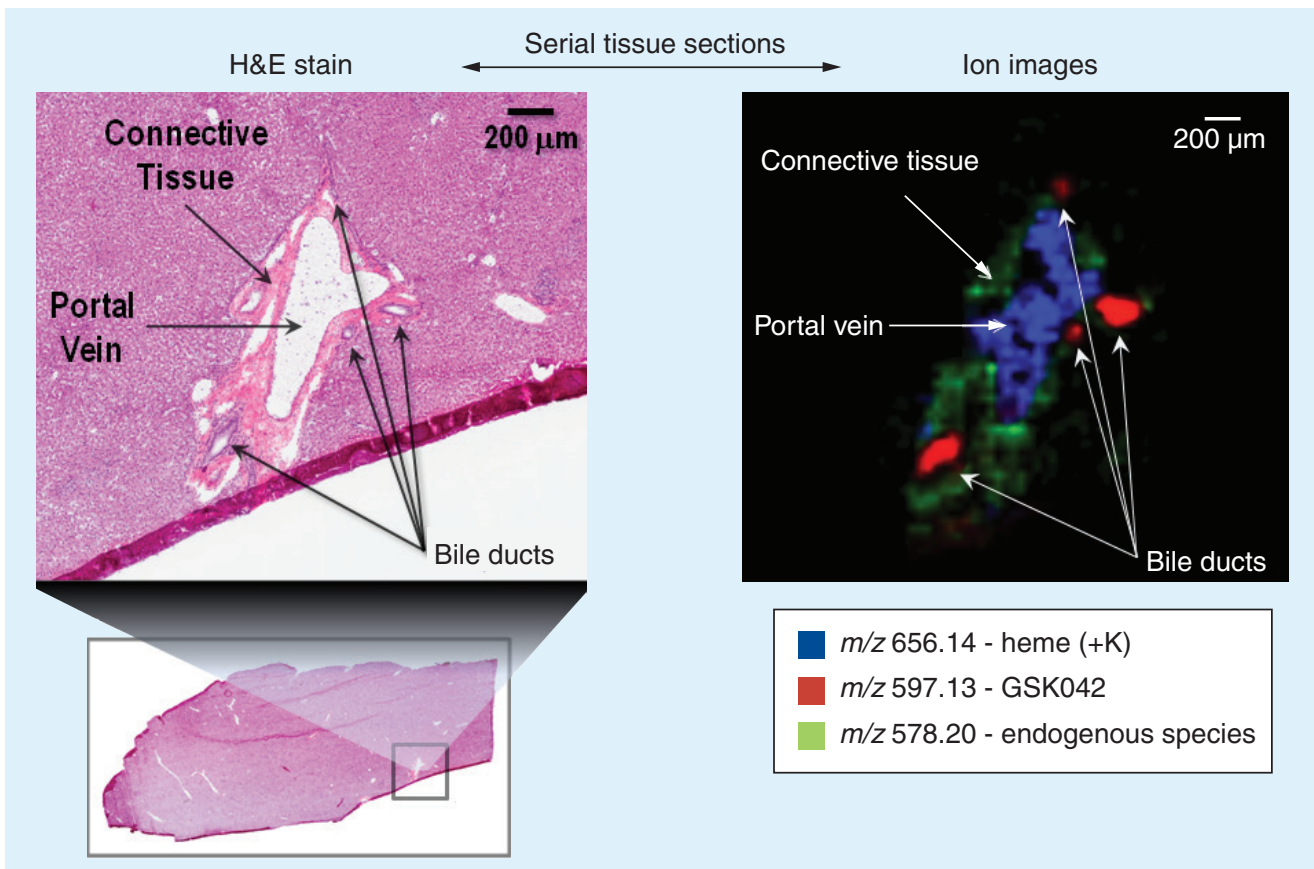

Figure 10. Correlation of histology and ion image in dog liver sections. A small area of the histology section is magnified showing details of structural features. A composite ion map (50 $\mu m$ spatial resolution), which corresponds to the same zoomed region, is constructed showing heme ions (potassium adduct) in the portal vein, a lapatinib metabolite (GSK042) in the bile ducts, and surrounding area from on endogenous species.

H\&E: Hematoxylin and eosin.

resolution, further differentiation of the kidney morphology can be resolved through IMS of endogenous lipids as well as dosed drugs [60].

One of the direct consequences of high lateral resolution imaging experiments in combination with high spectral resolution is the resultant large data sets which may require expansive data storage options. It is not uncommon to conduct an IMS experiment where $>10,000$ spectra are acquired, and when using an FT-ICR MS, each of these files can be several megabytes in size leading to datasets totaling $>50$ gigabytes. In addition, high performance computers (4; 8 -processors) with enhanced memory read/ write capabilities may be needed to process and manipulate these datasets efficiently.

In the practical application of IMS in drug development high lateral resolution is not always required. Rather, selection of the optimum spatial resolution should be based on tissue type, drug and metabolite concentration, MALDI ionization efficiency and the level of histology correlation necessary to answer key questions. In many ways the consideration of spatial resolution in IMS is similar to how we use Google Earth ${ }^{\circledR}$ (FIGURE I3). A low spatial resolution image covers much more area but with less detail while a zoom can provide greater detail but over a smaller area. The pragmatic application of IMS in preclinical applications will typically involve initial tissue section surveys at lower spatial resolution followed by higher spatial resolution images over selected tissue areas, potentially directed by histology analysis.

\section{Conclusion}

MALDI imaging is an emerging tool for enhancing our understanding of drug and metabolite tissue distributions. The ability to differentiate parent drug and metabolites in the same experiment without the need for labeling sets it apart from other methods. The impact of MALDI imaging in drug development stems from the unique ability of this method to correlate chemistry and biology in preclinical models. It provides the opportunity to carefully link tissue histology and drug and metabolite ion maps at meaningful levels of spatial resolution. The ability to map pharmacological receptors to drug or metabolite distribution is transformational in drug development. New insights into the mechanisms of pharmacology and toxicology will be possible. Furthermore, the tissue imaging data will impact associated 


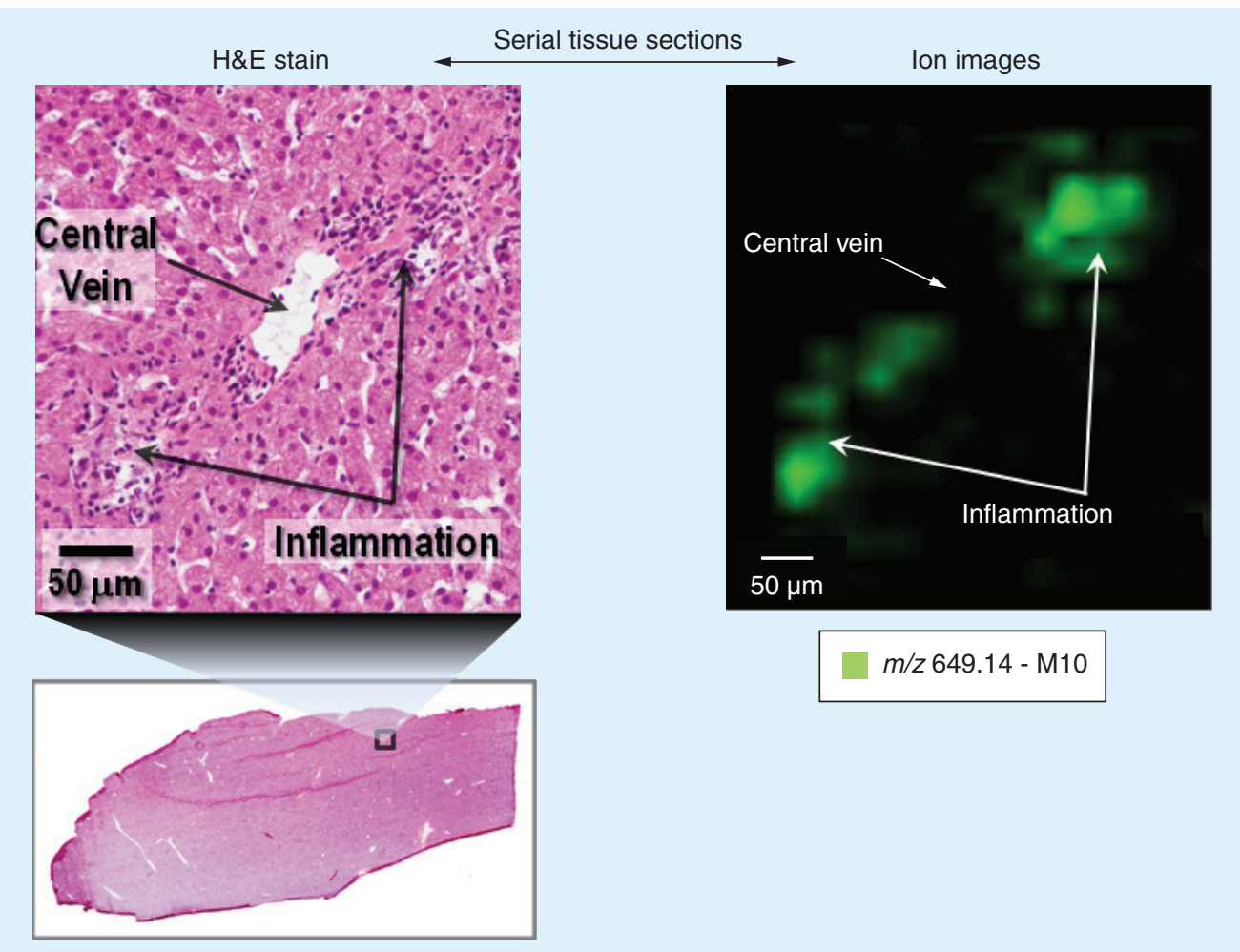

Figure 11. Correlation of histology and ion image in dog liver sections. A small area of the histology section is magnified showing regions of inflammation. An ion map (50 $\mu \mathrm{m}$ spatial resolution), which corresponds to the same zoomed region, shows the localization of lapatinib metabolite M10, localized only in the regions associated with the inflammation.

H\&E: Hematoxylin and eosin.

areas of physiologically based pharmacokinetic modeling as well as drug-drug interactions and transporters.

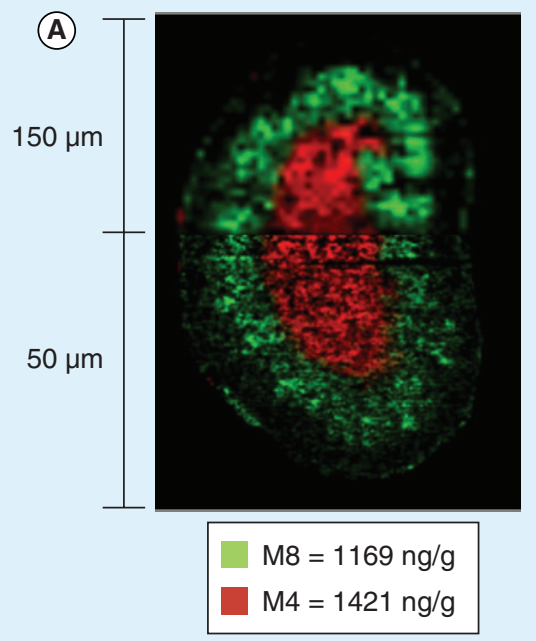

(B)

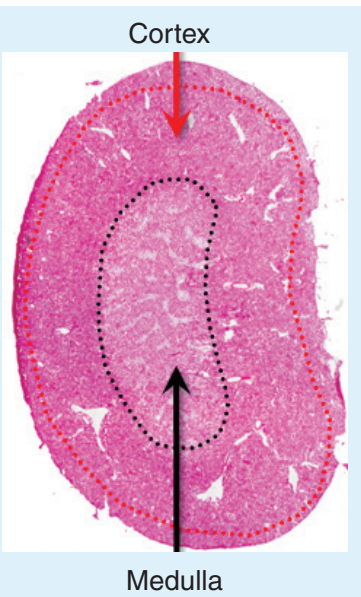

Medulla
Figure 12. Differential metabolite distributions in mouse kidney at $2 \mathbf{~ h}$. (A) Ion images for two mouse kidney metabolites; (top) $150 \mu \mathrm{m}$ spatial resolution (bottom) same section at $50 \mu \mathrm{m}$ resolution. (B) Hematoxylin and eosin stain of serial section.

\section{Future perspective}

The ability of MALDI imaging to provide a greater understanding of drug and metabolite tissue distribution is very exciting and at the heart of the widespread interest in this technique. Linking biology through histology and the intrinsic chemical properties of drugs and their metabolites could be translational in drug development. However, we must not let the early success of IMS in drug tissue distributions studies obscure the need for greater understanding of the science behind MALDI imaging. Collecting meaningful data requires careful experimental execution and an in-depth knowledge of the processes from sample preparation to data analysis. Over the next 5 years, a greater understanding of the MALDI experiment will help guide practitioners in the successful application of IMS to tissue distribution studies in drug development. In addition, venders and researchers will (continue to further develop and refine hardware and software to make the IMS experiment more robust and easier to implement. Researchers will also continue to develop and explore complimentary ionization methods that will broaden 
the scope of the IMS experiment. If we do not get ahead of ourselves, and continue to explore and develop the science associated with imaging MS, in 10 years time this methodology could be as commonplace as LC-MS is in drug development today.

\section{Financial \& competing interests disclosure The authors wish to thank GlaxoSmithKline colleagues: William Hardesty, Gary Bowers, Stephanie North, Gordon Dear, Claire Beaumont and Donna Fraser for helpful sug- gestions and review of the manuscript. The authors have no other relevant affliations or financial involvement with any organization or entity with a financial interest in or financial conflict with the subject matter or materials discussed in the manuscript apart from those disclosed. \\ No writing assistance was utilized in the production of this manuscript.}

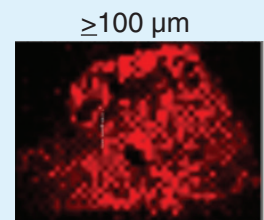

- Cover whole tissue

- Hot spots

- Good sensitivity

- Similar to whole-body autoradiography

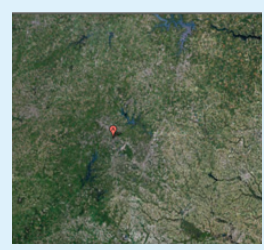

$<100->50 \mu \mathrm{m}$

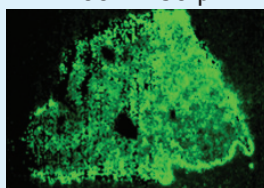

- Large portion tissue

- Some gross tissue features - Histology overlays

- Compares distributions

- Medium sensitivity

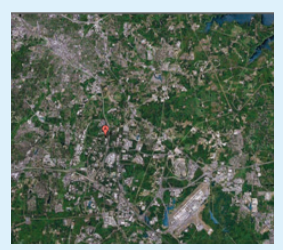

$\leq 50 \mu \mathrm{m}$

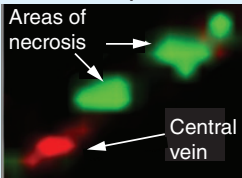

- Small portion of tissue

- Explore mechanisms

- Compare distributions

- Low sensitivity

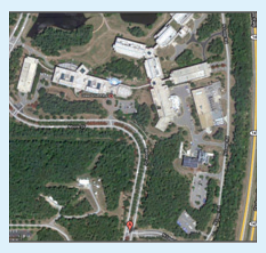

Figure 13. Impact of varying spatial resolution.

\section{Executive summary}

MALDI imaging MS is emerging as a powerful tool for gaining a more complete picture of drug and metabolite tissue distribution in preclinical drug development.

- Hardware and software developments will continue to refine and simplify MALDI imaging.

- Strategies and methods for the quantification of imaging MS data will continue to be a focal point of development.

- Current high-resolution mass spectrometers coupled with lasers capable of high spatial resolution $(<50 \mu \mathrm{m})$ permit MALDI images to be superimposed on histology sections taken by serial sectioning.

- This latter capability highlights the uniqueness of this technique to bridge biology and chemistry in the same experiment.

\section{Bibliography}

Papers of special note have been highlighted as:

- of interest

- of considerable interest

1 Marko-Varga G, Fehniger TE, Rezeli M et al. Drug localization in different lung cancer phenotypes by MALDI mass spectrometry imaging. J. Proteomics 74(7), 982-992 (2011).

2 Lanao JM, Fraile MA. Drug tissue distribution: study methods and therapeutic implications. Curr. Pharm. Des. 11(29), 3829-3845 (2005).

-. Comparitive analysis of tissue distributions with an emphasis on noninvasive methods in human studies. Also examines pharmacokinetic strategies for tissue distribution analyses.

3 Langer O, Muller M. Methods to assess tissue-specific distribution and metabolism of drugs. Curr. Drug Metab. 5(6), 463-481 (2004).

4 Monro AM. Interspecies comparisons in toxicology: the utility and futility of plasma concentrations of the test substance. Regul. Toxicol. Pharmacol. 12(2), 137-160 (1990).
5 Mouton JW, Theuretzbacher U, Craig WA et al. Tissue concentrations: do we ever learn? J. Antimicrob. Chemother. 61(2), 235-237 (2008).

6 Ryan DM, Cars O, Hoffstedt B. The use of antibiotic serum levels to predict concentrations in tissues. Scand. J. Infect. Dis. 18(5), 381-388 (1986).

7 Pellegatti M, Pagliarusco S. Drug and metabolite concentrations in tissues in relationship to tissue adverse findings: a review. Expert Opin. Drug Metab. Toxicol. 7(2), 137-146 (2011).

- Critical review assessing the value of preclinical drug and metabolite tissue concentrations and the potential risk of metabolite accumulation.

8 Solon EG, Schweitzer A, Stoeckli M et al. Autoradiography, MALDI-MS and SIMS-MS imaging in pharmaceutical discovery and development. AAPS J. 12(1), 11-26 (2010).

- Comprehensive review of molecular imaging techniques used to study drug tissue distributions.

9 Solon EG. Autoradiography: high-resolution molecular imaging in pharmaceutical discovery and development. Expert Opin. Drug Discov. 2(4), 503-514 (2007).

10 Stumpf WE. Drug localization and targeting with receptor microscopic autoradiography. J. Pharmacol. Toxicol. Methods 51(1), 25-40 (2005).

11 Sugiura Y, Setou M. Imaging mass spectrometry for visualization of drug and endogenous metabolite distribution: toward in situ pharmacometabolomes. J. Neuro.Pharmacol. 5(1), 31-43 (2010).

12 Khatib-Shahidi S, Andersson M, Herman JL et al. Direct molecular analysis of whole-body animal tissue sections by imaging MALDI mass spectrometry. Anal. Chem. 78(18), 6448-6456 (2006).

13 Stoeckli M, Staab D, Schweitzer A. Compound and metabolite distribution measured by MALDI mass spectrometric imaging in whole-body tissue sections. Int J. Mass Spec. 260 (2-3), 195-202 (2006).

14 Heeren RM, Smith DF, Stauber J et al. Imaging mass spectrometry: hype or hope? J. Am. Soc.Mass Spectrom. 20(6), 1006-1014 (2009). 
- Discussion of the parameters that define and control the implications, challenges, opportunities and (im) possibilities associated with the application of imaging MS.

15 Seeley EH, Caprioli RM. MALDI imaging mass spectrometry of human tissue: method challenges and clinical perspectives. Trends Biotechnol. 29(3), 136-143 (2011).

16 Schwamborn K, Caprioli RM. MALDI imaging mass spectrometry - painting molecular pictures. Mol. Oncol. 4(6), 529-538 (2010).

17 Schwamborn K, Caprioli RM. Molecular imaging by mass spectrometry - looking beyond classical histology. Nat. Rev. Cancer 10(9), 639-646 (2010).

18 Svatos A. Mass spectrometric imaging of small molecules. Trends Biotechnol. 28(8), 425-434 (2010).

19 Goodwin RJ, Pitt AR. Mass spectrometry imaging of pharmacological compounds in tissue sections. Bioanalysis 2(2), 279-293 (2010).

20 Pol J, Strohalm M, Havlicek V et al. Molecular mass spectrometry imaging in biomedical and life science research. Histochem. Cell Biol. 134(5), 423-443 (2010).

21 Amstalden van Hove ER, Smith DF, Heeren RM. A concise review of mass spectrometry imaging. J. Chromatogr. A 1217(25), 3946-3954 (2010).

22 Vickerman JC. Molecular imaging and depth profiling by mass spectrometry - SIMS, MALDI or DESI? Analyst 136(11), 2199-2217 (2011).

23 Goodwin RJ, Pitt AR. Mass spectrometry imaging of pharmacological compounds in tissue sections. Bioanalysis 2(2), 279-293 (2010).

- Comprehensive review of MS-based imaging technologies for the analysis of drug distributions.

24 Greer T, Sturm R, Li L. Mass spectrometry imaging for drugs and metabolites. J. Proteomics doi:10.1016/j.jprot.2011.03.032 (2011) (Epub ahead of print).

25 Rubakhin SS, Sweedler JV. A mass spectrometry primer for mass spectrometry imaging. Methods Mol. Biol. 656, 21-49 (2010).

26 Knochenmuss R. Ion formation mechanisms in UV-MALDI. Analyst 131(9), 966-986 (2006).

- In-depth review of the theories and mechanisms describing ion formation in MALDI.
27 Chaurand P, Cornett DS, Angel PM et al. From whole-body sections down to cellular level, multiscale imaging of phospholipids by MALDI mass spectrometry. Mol. Cell Proteomics 10(2), O110.004259 (2011).

28 Lemaire R, Desmons A, Tabet JC et al. Direct analysis and MALDI imaging of formalinfixed, paraffin-embedded tissue sections. J. Proteome. Res. 6(4), 1295-1305 (2007).

29 Groseclose MR, Massion PP, Chaurand P et al. High-throughput proteomic analysis of formalin-fixed paraffin-embedded tissue microarrays using MALDI imaging mass spectrometry. Proteomics 8(18), 3715-3724 (2008).

30 Drexler DM, Garrett TJ, Cantone JL et al. Utility of imaging mass spectrometry (IMS) by matrix-assisted laser desorption ionization (MALDI) on an ion trap mass spectrometer in the analysis of drugs and metabolites in biological tissues. J. Pharmacol. Toxicol. Methods 55(3), 279-288 (2007).

31 Strohalm M, Strohalm J, Kaftan F et al. Poly[N-2hydroxypropyl)methacrylamide]based tissue-embedding medium compatible with MALDI mass spectrometry imaging experiments. Anal. Chem. 83(13), 5458-5462 (2011).

32 Dreisewerd $\mathrm{K}$. The desorption process in MALDI. Chem. Rev. 103(2), 395-426 (2003).

33 Horneffer V, Forsmann A, Strupat K et al. Localization of analyte molecules in MALDI preparations by confocal laser scanning microscopy. Anal. Chem. 73(5), 1016-1022 (2001).

34 Knochenmuss R, Zenobi R. MALDI ionization: the role of in-plume processes. Chem. Rev. 103(2), 441-452 (2003).

35 Knochenmuss R. Photoionization pathways and free electrons in UV-MALDI. Anal. Chem. 76(11), 3179-3184 (2004).

36 Knochenmuss R, Zhigilei LV. Molecular dynamics simulations of MALDI: laser fluence and pulse width dependence of plume characteristics and consequences for matrix and analyte ionization. J. Mass Spectrom. 45(4), 333-346 (2010).

37 Hawkridge AM, Muddiman DC. Mass spectrometry-based biomarker discovery: toward a global proteome index of individuality. Annu. Rev. Anal. Chem. (Palo. Alto.Calif.) 2, 265-277 (2009).

38 Jaskolla T, Karas M. Compelling evidence for lucky survivor and gas phase protonation: the unified MALDI analyte protonation mechanism. J. Am.Soc. for Mass Spectrom. 22(6), 976-988 (2011)
39 Aerni HR, Cornett DS, Caprioli RM. Automated acoustic matrix deposition for MALDI sample preparation. Anal. Chem. 78(3), 827-834 (2006).

40 Murphy RC, Hankin JA, Barkley RM et al. MALDI imaging of lipids after matrix sublimation/deposition. Biochim. Biophys. Acta DOI:10.1016/j.bbalip.2011.06.019 (2011) (Epub ahead of print).

41 Hankin JA, Barkley RM, Murphy RC. Sublimation as a method of matrix application for mass spectrometric imaging. J. Am. Soc. Mass Spectrom. 18(9), 1646-1652 (2007).

42 Chaurand P, Schwartz SA, Capriolo RM. Profiling and imaging proteins in tissue sections by MS. Anal. Chem. 76(5), 87A-93A (2004).

43 Deininger SO, Cornett DS, Paape R et al. Normalization in MALDI-TOF imaging datasets of proteins: practical considerations. Anal. Bioanal. Chem. 401(1), 167-181 (2011).

44 Bonnel D, Legouffe R, Willand et al. MALDI imaging techniques dedicated to drugdistribution studies. Bioanalysis 3(12), 1399-1406 (2011).

45 Borgaonkar SP, Hocker H, Shin H et al. Comparison of normalization methods for the identification of biomarkers using MALDI-TOF and SELDI-TOF mass spectra. OMICS 14(1), 115-126 (2010).

46 Deininger SO, Cornett DS, Paape R et al. Normalization in MALDI-TOF imaging datasets of proteins: practical considerations. Anal. Bioanal. Chem. 401(1), 167-181 (2011).

47 Meuleman W, Engwegen JY, Gast MC, et al. Comparison of normalisation methods for surface-enhanced laser desorption and ionisation (SELDI) time-of-flight (TOF) mass spectrometry data. BMC Bioinformatics 9, 88 (2008).

48 Reyzer ML, Hsieh Y, Ng K et al. Direct analysis of drug candidates in tissue by matrix-assisted laser desorption/ionization mass spectrometry. J. Mass Spectrom. 38(10), 1081-1092 (2003).

49 Koeniger SL, Talaty N, Luo Y et al. A quantitation method for mass spectrometry imaging. Rapid Commun. Mass Spectrom. 25(4), 503-510 (2011).

50 Yamada Y, Hidefumi K, Shion H et al. Distribution of chloroquine in ocular tissue of pigmented rat using matrix-assisted laser desorption/ionization imaging quadrupole time-of-flight tandem mass spectrometry. Rapid Commun. Mass Spectrom. 25(11), 1600-1608 (2011). 
51 Hu Q, Noll RJ, Li H et al. The Orbitrap: a new mass spectrometer. J. Mass Spectrom. 40(4), 430-443 (2005).

52 Perry RH, Cooks RG, Noll RJ. Orbitrap mass spectrometry: instrumentation, ion motion and applications. Mass Spectrom. Rev. 27(6), 661-699 (2008).

53 Marshall AG, Hendrickson CL, Jackson GS. Fourier transform ion cyclotron resonance mass spectrometry: a primer. Mass Spectrom. Rev. 17(1), 1-35 (1998).

54 Bristow AW. Accurate mass measurement for the determination of elemental formula - a tutorial. Mass Spectrom. Rev. 25(1), 99-111 (2006).

55 Zhang H, Zhu M, Ray KL et al. Mass defect profiles of biological matrices and the general applicability of mass defect filtering for metabolite detection. Rapid Commun. Mass Spectrom. 22(13), 2082-2088 (2008)

56 Zhang H, Zhang D, Ray K et al. Mass defect filter technique and its applications to drug metabolite identification by high-resolution mass spectrometry. J. Mass Spectrom. 44(7), 999-1016 (2009).

57 Zhang D, Cheng PT, Zhang H. Mass defect filtering on high resolution LC-MS data as a methodology for detecting metabolites with unpredictable structures: identification of oxazole-ring opened metabolites of muraglitazar. Drug Metab. Lett. 1(4), 287-292 (2007).
58 Hopfgartner G, Varesio E, Stoeckli M. Matrix-assisted laser desorption/ionization mass spectrometric imaging of complete rat sections using a triple quadrupole linear ion trap. Rapid Commun. Mass Spectrom. 23(6), 733-736 (2009).

59 Prideaux B, Dartois V, Staab D et al. High-sensitivity MALDI-MRM-MS imaging of moxifloxacin distribution in tuberculosisinfected rabbit lungs and granulomatous lesions. Anal. Chem. 83(6), 2112-2118 (2011)

60 Rompp A, Guenther S, Takats Z et al. Mass spectrometry imaging with high resolution in mass and space (HR(2) MSI) for reliable investigation of drug compound distributions on the cellular level. Anal. Bioanal. Chem. 401(1), 65-73 (2011)

- Combines high mass resolution and high spatial resolution in drug imaging experiments from several tissues.

61 McLean JA, Fenn LS, Enders JR. Structurally selective imaging mass spectrometry by imaging ion mobility-mass spectrometry. Methods Mol. Biol. 656, 363-383 (2010).

62 McLean JA, Ridenour WB, Caprioli RM. Profiling and imaging of tissues by imaging ion mobility-mass spectrometry. J. Mass Spectrom. 42(8), 1099-1105 (2007).

63 Kanu AB, Dwivedi P, Tam M et al. Ion mobility-mass spectrometry. J. Mass Spectrom. 43(1), 1-22 (2008)
64 Woods AS, Jackson SN. The application and potential of ion mobility mass spectrometry in imaging MS with a focus on lipids. Methods Mol. Biol. 656, 99-111 (2010).

65 Chaurand P, Schriver KE, Caprioli RM. Instrument design and characterization for high resolution MALDI-MS imaging of tissue sections. J. Mass Spectrom. 42(4), 476-489 (2007).

66 Jurchen JC, Rubakhin SS, Sweedler JV. MALDI-MS imaging of features smaller than the size of the laser beam. J. Am. Soc. Mass Spectrom. 16(10), 1654-1659 (2005).

\section{- Websites}

101 Specialized Scientific Instrumentation for Molecular Imaging. http://htximaging.com/Content. aspx? type $=$ TMS

102 A new matrix application device for MALDI tissue imaging. www.maldi-msi.org/download/tech18_ ImagePrep.pdf

103 Core technology for sample processing used in MS tissue imaging and profiling. www.ssi.shimadzu.com/products/literature/ Biotech/CHIP-1000.pdf

104 Labcyte. www.labcyte.com/Portrait\%C2\%AE_630 Spotter/Default.92.html 